\title{
A Neural Mechanism Underlying Memory Failure in Older Adults
}

\author{
W. Dale Stevens, ${ }^{1,2,4}$ Lynn Hasher, ${ }^{2,4}$ Kimberly S. Chiew, ${ }^{2}$ and Cheryl L. Grady ${ }^{2,3,4}$ \\ ${ }^{1}$ Department of Psychology, Harvard University, Cambridge, Massachusetts 02138, ${ }^{2}$ Rotman Research Institute at Baycrest, and Departments of 3 Psychiatry \\ and ${ }^{4}$ Psychology, University of Toronto, Toronto, Ontario, Canada M5S 3G3
}

Older adults have reduced memory, primarily for recall, but also for recognition (Craik and McDowd, 1987), particularly for unfamiliar faces (Bartlett et al., 1989). Behavioral studies have shown that age-related memory declines are due in part to distraction from impaired inhibition of task-irrelevant input during encoding (Healey et al., 2008). Functional magnetic resonance imaging (fMRI) has been used to uncover the sources of memory deficits associated with aging. To date, this work has focused on successful encoding, while the neural correlates of unsuccessful encoding are unknown. Here, we provide novel evidence of a neural mechanism underlying memory failures exclusively affecting older adults. Whereas both younger and older adults showed reduced activation of brain regions important for encoding (e.g., hippocampus) during unsuccessful encoding, only older adults showed increased activity in brain regions mediating distraction (e.g., auditory cortex) and in left prefrontal cortex. Further, these regions were functionally connected with medial parietal areas, previously identified as default mode regions (Raichle and Snyder, 2007), which may reflect environmental monitoring. Our results suggest that increased distraction from task-irrelevant input (auditory in this case), associated with the unfamiliar and noisy fMRI environment, may increase environmental monitoring. This in turn could hinder suppression of default mode processing, resulting in memory failures in older adults. These findings provide novel evidence of a brain mechanism underlying the behavioral evidence that impaired inhibition of extraneous input during encoding leads to memory failure in older adults and may have implications for the ubiquitous use of fMRI for investigating neurocognitive aging.

Key words: cognitive aging; memory; fMRI; distraction; functional connectivity; default mode

\section{Introduction}

Event-related functional magnetic resonance imaging (fMRI) allows us to examine the processes involved in successful versus unsuccessful memory events. To date, research has focused on brain activity associated with successful memory in older adults (Daselaar et al., 2003; Morcom et al., 2003; Miller et al., 2008), while neural correlates of unsuccessful encoding resulting in memory failures are unknown. However, it is just as important, if not more so, to understand activity specifically associated with age-related memory failures, an approach taken here.

One possible cause of memory failures is the insufficient engagement of brain regions important for encoding new information [e.g., medial temporal lobe (MTL) regions]. Increased activity in MTL regions during encoding of specific items has been associated with subsequent ability of both younger (Brewer et al., 1998) and older adults (Morcom et al., 2003) to recognize those items, and reduced hippocampal activity has been reported in

Received June 5, 2008; revised July 31, 2008; accepted 0ct. 20, 2008.

This work was supported by Canadian Institutes of Health Research Grant MOP14036 to C.L.G., a Doctoral Award to W.D.S., and National Institute on Aging Grant R37 AG04306 to L.H.C.L.G. is also supported by the Canada Research Chairs program, the Ontario Research Fund, and the Canadian Foundation for Innovation. We thank the MRI technologists at Sunnybrook Health Sciences Centre, Toronto, Ontario, Canada, for assistance with the experiment.

Correspondence should be addressed to Dr. W. Dale Stevens, Department of Psychology, Harvard University, 33 Kirkland Street, Cambridge, MA 02138.E-mail: dstevens@wjh.harvard.edu.

DOI:10.1523/JNEUROSCI.2622-08.2008

Copyright $\odot 2008$ Society for Neuroscience $\quad$ 0270-6474/08/2812820-05\$15.00/0 older adults, relative to younger adults, during encoding (Grady et al., 1995).

However, another possible cause of age-related encoding failures is distraction. Older adults are more vulnerable to distracting effects of task-irrelevant stimuli (Connelly et al., 1991; Lustig et al., 2006; Healey et al., 2008). There is evidence of age-related performance declines on some tasks during fMRI scanning, compared with performance outside of the scanner (Gutchess and Park, 2006; Grady et al., 2008), suggesting that the noisy fMRI environment has disproportionately detrimental effects on older adults' performance. In addition, default mode regions (DMRs), a network of brain regions consistently showing increased activity during rest relative to a wide range of active tasks (Raichle and Snyder, 2007), are typically "deactivated" during most externally focused cognitive tasks in healthy younger adults, whereas older adults fail to suppress activity in DMRs (Lustig et al., 2003; Grady et al., 2006; Miller et al., 2008). One theory suggests that DMRs are involved in "surveillance of the internal and external environments" (Gusnard et al., 2001), potentially including, for example, "attending to scanner noise and incidental light" (Gilbert et al., 2007). The effects of distraction during encoding might then be seen in the brain as increased activity in both auditory cortex and DMRs during failed encoding. It may be that both distractibility and reduced engagement of memory-related areas contribute to memory failures in older adults.

Here, we examined brain activity leading to successful and 
unsuccessful subsequent recognition of unfamiliar faces in younger and older adults using fMRI. Brain activity during the first presentations of faces was assessed, with these events categorized by the recognition response given at the second presentation. We hypothesized that during encoding of faces subsequently forgotten, relative to those later remembered, older adults would show less activity in MTL regions, and more activity in areas that process task-irrelevant stimuli and internal thoughts, such as auditory cortex and DMRs, respectively. We further expected that the effects of distraction in the brain during encoding would be seen only in older adults, consistent with age-related differences in ability to suppress irrelevant information during working memory tasks (Gazzaley et al., 2005).

\section{Materials and Methods}

Participants. Participants were 24 healthy adults, 12 younger (mean age $\pm \mathrm{SD}=26.4 \pm 3.8$ years, range $=22-36 ; 5$ female) and 12 older (mean age $\pm \mathrm{SD}=70.2 \pm 4.1$ years; range $=64-78,8$ female). None of the participants had any history of psychiatric, neurological, or other medical illness, or history of drug or alcohol abuse, which might compromise cognitive function. All participants were right handed and scored in the normal range on the Mini Mental Status Exam $(>26)$ (Folstein et al., 1975). The young adults had more years of education than the older adults (17.8 vs 13.6 years, $t_{(20)}=3.2, p<0.01$ ), but there were no differences between groups on vocabulary scores [Mill Hill Vocabulary Test (Raven et al., 1988)] or verbal recognition performance [Hopkins Verbal Learning Test (Brandt, 1991)]. Two older adults were excluded from analysis due to insufficient numbers of responses; thus, 10 older participants were included in all analyses $(70.3 \pm 4.5$ years; range $=$ $64-78,7$ female). All procedures were approved by the ethics committees of Baycrest Centre and Sunnybrook Health Sciences Centre, Toronto, Ontario, Canada.

Experimental design. Participants were scanned using fMRI while viewing grayscale images of unfamiliar faces in a continuous recognition paradigm. Stimuli consisted of grayscale images of unfamiliar faces presented on a black background (Haxby et al., 1996). For all "face-trials," a face was presented in the center of the field of view for $1 \mathrm{~s}$, followed by a $3 \mathrm{~s}$ fixation cross. For all "null-trials," a fixation cross was presented for 4 s. Stimuli were presented via MRI compatible goggles (Silent Vision SV-4021; Avotec) mounted on a head coil above the participants' eyes. Participants made responses using MRI compatible response boxes (Rowland USB Response Box).

A mixed block/event-related design was used, with 3 different blocktypes: fixation (FIX), memory task (MEM), and control task (CON). Each block began with a one word instruction cue for the block (4s) followed by a fixation-cross (4 s). Within each task block (i.e., MEM and $\mathrm{CON}$ ), face trials and null trials were presented in a standard stochastic event-related distribution. The experiment consisted of six functional runs $($ duration $=520 \mathrm{~s})$, each with 1 FIX-block, 1 CON-block, and 2 MEM-blocks, presented in random order. The current analyses used only the MEM-blocks, during which participants performed a continuous face-recognition task. For these blocks, 180 face stimuli were used, 120 of which were randomly selected and presented a second time, after which 60 of these were randomly selected for a third presentation (repeated items were not used for the current analysis). All faces were presented in random order.

For each face, participants made a recognition judgment, indicating whether or not they had seen the face previously in the experiment (new or old), and their level of confidence (high or low) in their decision. This was indicated with a single four-alternative forced-choice response (high-confident new, low-confident new, low-confident old, or highconfident old); using the left middle finger, left index finger, right index finger, and right middle finger, respectively, for all participants. First presentations of faces $(n=120,60$ female) were classified as encoding trials, and categorized into three different trial-types based on the recognition response to the second presentation of each face: (1) faces subsequently forgotten (misses), collapsed across high and low confidence to obtain sufficient numbers of trials; (2) faces subsequently remembered with high confidence (HC-hits); and (3) faces subsequently remembered with low confidence (LC- hits).

fMRI data acquisition and analysis. Participants were scanned using a whole-body 3.0 T MR scanner (Signa 3T hardware, VH3M4 software; GE Healthcare) with a standard quadrature birdcage head coil. Functional scans were obtained by using a single-shot $\mathrm{T} 2{ }^{\star}$-weighted pulse sequence $\left(\mathrm{TR}=2000 \mathrm{~ms}, \mathrm{TE}=40 \mathrm{~ms}\right.$, flip angle $70^{\circ}, \mathrm{FOV}=20 \mathrm{~cm}, 64 \times 64$ acquisition matrix) with spiral in/out acquisition, and consisting of 26 contiguous, 5-mm-thick axial slices. In addition, we obtained a T-1 weighted structural MRI using a standard 3D fast-spoiled gradient-echo pulse sequence $(\mathrm{TR}=7.0 \mathrm{~ms}, \mathrm{TE}=3.1 \mathrm{~ms}$, inversion recovery preparation time $=300 \mathrm{~ms}$, flip angle $15^{\circ}, 22 \times 16.5$ field of view, $256 \times 192$ acquisition matrix, 124 axial slices, $1.4 \mathrm{~mm}$ thick).

The fMRI data were processed using Analysis of Functional Neuroimages (AFNI) (Cox and Hyde, 1997) and SPM99 (Friston et al., 1995). In AFNI, time series data were spatially coregistered to correct for head motion using a 3D Fourier transform interpolation, using a functional volume that minimized the amount of motion to $<2 \mathrm{~mm}$. Then, slicetiming correction was performed. Linear detrending of the data was implemented for each run separately. Last, each participant's images were spatially normalized to Montreal Neurological Institute (MNI) space and smoothed with an $8 \mathrm{~mm}$ Gaussian filter using SPM99. The resulting voxel size was $4 \times 4 \times 4 \mathrm{~mm}$.

Spatiotemporal Partial Least Squares (PLS), a multivariate analysis tool (McIntosh et al., 2004), was used to analyze the fMRI data. PLS operates on the covariance between brain voxels [blood-oxygen-level dependent (BOLD) signal] and the experimental design to identify a new set of variables (so-called latent variables or LVs) that optimally relate the two sets of measurements. Each LV contains a spatial activity pattern depicting the brain regions that, as a whole, show the strongest relation to (i.e., are covariant with) the task contrast(s) identified by the LV, in this case, failed versus successful encoding events. The analysis included 8 poststimulus TRs (16s) for each event and activity at each time point was normalized to activity in the first TR of the trial. PLS, as applied to event-related data, results in a set of brain regions that are reliably related to the task contrasts for each TR on each LV (McIntosh et al., 2004). Each brain voxel has a weight, known as a salience, which is proportional to the covariance of activity with the task contrast at each time point on each LV. Multiplying the BOLD signal value in each brain voxel for each subject by the salience for that voxel, and summing across all voxels, gives a "brain" score for each subject at each TR on a given LV. The significance for each LV as a whole was determined by using a permutation test (McIntosh et al., 1996). Additionally, the reliability of the saliences for the brain voxels characterizing each pattern identified by the LVs was determined via a bootstrap estimation of the SEs (Efron and Tibshirani, 1986). Peak voxels with a salience/SE ratio $>3.0$ were considered to be reliable (Sampson et al., 1989). Clusters containing at least 10 reliable voxels were extracted, and a local maximum for each cluster was defined as the voxel with a salience/SE ratio higher than any other voxel in a $2 \mathrm{~cm}$ cube centered on that voxel. Coordinates of these locations are reported in MNI space.

To contrast brain activity associated with unsuccessful versus successful encoding in the two groups, PLS was performed on events for the three trial-types described above (misses, LC-hits, HC-hits) for both groups simultaneously. This analysis revealed a single significant LV $(p=0.03)$ that identified a pattern of brain activity that differentiated subsequent misses from both HC-hits and LC-hits in older adults, but did not reliably differentiate any of the conditions in the younger adults. Hence, for assessing activity in specified voxels [i.e., region of interest (ROI) analyses], trials for subsequently remembered faces were collapsed across confidence (i.e., hits, collapsed across low and high confidence). We then extracted the peak signal change from these regions and compared these values between successful and unsuccessful encoding and between young and older adults using ANOVA. To identify functionally connected networks of brain regions associated with failed and successful encoding, "seed" PLS (McIntosh, 1999; Grady et al., 2003) was used. Seed PLS correlates activity in a ROI, identified in the original task PLS, with 
activity in all other brain voxels to assess the functional connectivity of this region.

\section{Results}

Older and younger adults showed no difference in the number of faces correctly recognized (i.e., LC-hits and HC-hits). However, older adults forgot more faces than younger adults (i.e., "misses"; $t_{(20)}=$ $2.8, p=0.02$ ) (Table 1 ). (For recognition responses to first presentations of faces, see supplemental Table 1, available at www.jneurosci.org as supplemental material). Based on the pattern of activity identified by PLS (LV: $p=0.03$ ) that reliably differentiated encoding of subsequently forgotten faces (misses) from subsequently remembered faces (both LC-hits and HC-hits) in the older adults, but not in younger adults, we examined activity in regions showing this pattern. Consistent with previous findings (Morcom et al., 2003), ROI analysis showed that successful encoding (LC-hits and HC-hits) was associated with increased activity in several regions in older adults, notably the hippocampus (Fig. 1a). Although the overall pattern of activity characterizing hits and misses was not reliable in the young group, activity in the hippocampus showed a similar increase in younger adults. This was confirmed by a 2 (group: old vs young) $\times 2$ (trial-type: forgotten vs remembered) ANOVA that showed a significant main effect of trial-type (forgotten vs remembered faces $)$ in the hippocampus $\left(F_{(1,20)}=7.1\right.$, $p=0.015)$, but no significant difference between the two age groups (group $\times$ trial-type interaction: $\mathrm{F}<1$ ).

However, consistent with the importance of age-related distraction, unsuccessful encoding only in the older adults was accompanied by increased activity in auditory cortex (Fig. 1b), as well as in left dorsolateral prefrontal cortex (PFC), right insula, and the right fusiform gyrus (Table 2 ). In contrast, young adults showed no difference in activity in these regions across face encoding trial-types (nor were there any regions with greater subsequent forgetting activity in younger adults relative to older adults). The age-related difference in activity of auditory cortex was confirmed via ANOVA as described above. There was a significant group by trial-type interaction for peak activity in both left auditory cortex $\left(F_{(1,20)}=5.3, p=0.03\right)$ and right auditory cortex $\left(F_{(1,20)}=6.2, p=0.02\right)$.

Given that older adults had more misses than younger adults, one could question whether the pattern of activity characterizing unsuccessful encoding in the older group was due to this behavioral difference. To address this question, we divided the younger group into two subgroups (low-performing and highperforming young adults) based on a median split of the number of misses to see if the low-performing subgroup would show the

(a)

(b)
Table 1. Performance on the face recognition task

\begin{tabular}{lllll}
\hline & Old & Young (all) & $\begin{array}{l}\text { Young (high- } \\
\text { performing) }\end{array}$ & $\begin{array}{l}\text { Young (low- } \\
\text { performing) }\end{array}$ \\
\hline Miss & $0.43 \pm 0.17$ & $0.26 \pm 0.10^{*}$ & $0.17 \pm 0.06^{*}$ & $0.35 \pm 0.05$ \\
Low confidence hit & $0.15 \pm 0.11$ & $0.24 \pm 0.11$ & $0.23 \pm 0.14$ & $0.25 \pm 0.08$ \\
High confidence hit & $0.42 \pm 0.17$ & $0.50 \pm 0.18$ & $0.60 \pm 0.19$ & $0.40 \pm 0.09$ \\
\hline
\end{tabular}

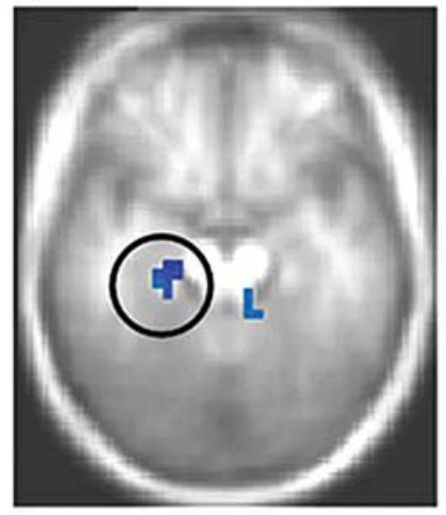

$Z=-20$

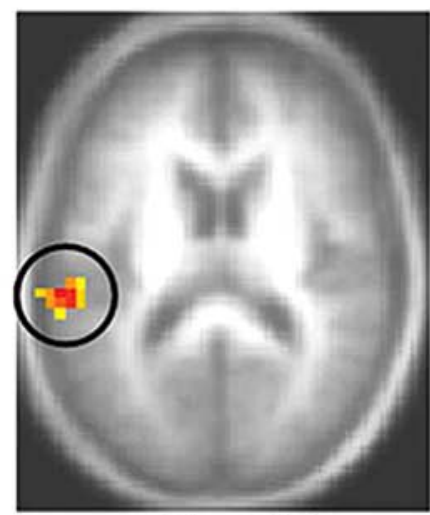

$Z=+12$

Figure 1. Brain activity for failed versus successful encoding in younger and older adults. $\boldsymbol{a}$, Area of left hippocampus (circled) with increased activity in older adults for faces subsequently remembered. Mean signal change versus baseline differs across conditions for both groups (see Table 2 for coordinates of regions). $\boldsymbol{b}$, Area of left auditory cortex (circled) with greater activity in older adults for faces subsequently forgotten. Mean signal change versus baseline differs across conditions only in older adults. Highlighted brain areas are shown on axial images from the average structural MRI of all 22 participants. Coordinate (Z) for each image is in MNI space. Error bars represent SE.

same pattern of brain activity, differentiating faces later forgotten versus those later remembered, seen in the older adults. This was not the case. Post hoc comparisons of means (Tukey's honestly significant difference) indicated that high-performing younger adults had significantly fewer misses than the older adults and marginally fewer misses than low-performing young adults ( $p=$ 0.002 and $p=0.057$, respectively), whereas low-performing young adults and older adults did not differ $(p=0.444)$ (Table 1). However, there were no regions where low-performing young adults had greater activity than high-performing young adults. Instead, the high-performing subgroup showed greater activity in bilateral extrastriate and MTL regions when viewing faces subsequently remembered (supplemental Fig. 1, available at www.j- 
Table 2. Brain regions showing subsequent memory effects in older adults

\begin{tabular}{|c|c|c|c|c|}
\hline Region & $x$ & $Y$ & $Z$ & Ratio \\
\hline \multicolumn{5}{|l|}{ Forgotten $>$ remembered } \\
\hline L frontal gyrus & -36 & 24 & 24 & 4.7 \\
\hline L temporal & -64 & -28 & 12 & 6.6 \\
\hline R temporal & 48 & -32 & 4 & 3.8 \\
\hline \multirow[t]{2}{*}{ R insula } & 32 & -16 & -12 & 5.8 \\
\hline & 40 & 0 & -8 & 5.4 \\
\hline R fusiform & 32 & -56 & -16 & 4.2 \\
\hline \multicolumn{5}{|l|}{ Remembered $>$ forgotten } \\
\hline L hippocampus & -24 & -20 & -20 & -7.0 \\
\hline L parietal & -60 & -60 & 44 & -6.1 \\
\hline Mid cingulate & -12 & -8 & 48 & -5.8 \\
\hline Dorsomedial prefrontal & -8 & 20 & 56 & -5.1 \\
\hline
\end{tabular}

(a)

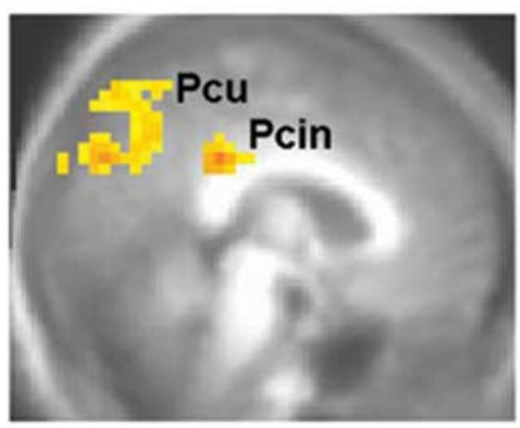

$x=-4$

Figure 2. Functional connectivity of auditory cortex in older adults during failed encoding. Regions functionally connected to bilateral auditory cortex are shown on medial $(\boldsymbol{a})$ and lateral $(\boldsymbol{b})$ sagittal images. Activity in all colored regions was positively correlated with activity in bilateral auditory cortex only in older participants for faces subsequently forgotten. Pcin, Posterior cingulate; Pcu, precuneus; STS, superior temporal sulcus; IFG, inferior frontal gyrus. Highlighted brain areas are shown on the average structural MRI of all 22 participants. Coordinate $(X)$ for each image is in MNI space.

neurosci.org as supplemental material). Thus, younger adults who forgot more faces did not show increased activity in auditory cortex when encoding these forgotten faces, suggesting that this pattern in older adults reflects susceptibility to distraction rather than a higher proportion of forgotten faces per se.

We then examined the functional connectivity of the auditory areas showing an age effect using a seed PLS, which correlated activity in each of the auditory regions with activity in all other brain voxels, and then examined how these correlation patterns differed across conditions and age groups. This analysis revealed a significant pattern of functional connectivity $(p=0.002)$ that was reliable for both auditory cortices (based on a 95\% confidence interval) only in older adults and only during encoding of faces later forgotten $(r=0.80$ and $r=0.50$ for the right and left auditory regions, respectively; $r$ values represent the correlation between activity in each seed region and the whole brain pattern of correlations identified by the seed PLS). Activity in the auditory regions was positively correlated with activity in bilateral PFC and temporal lobes, as well as bilateral inferior parietal regions. Importantly, the auditory cortices were functionally connected to medial parietal areas, including posterior cingulate and precuneus, previously identified as DMRs (Raichle et al., 2007) (Fig. 2a), in addition to a left PFC region, very similar to that seen in the task analysis (Fig. 2b).

\section{Discussion}

These analyses were designed to investigate the neural mechanisms underlying failed memory encoding in older adults and to determine if brain activity reflects the inability of older adults to suppress distraction from task-irrelevant stimuli (Healey et al., 2008). We used PLS analysis of fMRI data to identify those brain regions where activity differentiated failed versus successful encoding in older and younger adults. We then used seed PLS to examine the functional connectivity of auditory regions that showed increased activity during unsuccessful, relative to successful, encoding only in older adults.

The results of our task PLS analysis revealed reduced engagement of brain regions important for memory encoding (e.g., left hippocampus) during unsuccessful encoding in both younger and older adults, providing further support for the idea that MTL activity during encoding is predictive of successful memory in both young (Brewer et al., 1998) and old adults (Morcom et al., 2003). Critically, we observed additional regions showing increased activity associated with failed encoding in the older adults only, including left PFC and other regions that may reflect the processing of task-irrelevant but distracting sensory input (e.g., auditory regions) during encoding of faces subsequently forgotten. Further, the results of our seed PLS revealed that these regions were functionally connected to DMRs, which may reflect an inability to suppress environmental monitoring during the memory task. This finding is consistent with previous research indicating that older adults, and low-performing older adults in particular, failed to deactivate aspects of the default network (medial parietal regions) to the same extent as younger adults during encoding (Miller et al., 2008). Thus, both the increased activity in auditory regions during unsuccessful encoding and the functional connectivity of these regions are consistent with previous research showing that older adults have difficulty suppressing irrelevant auditory information (Fabiani et al., 2006) and irrelevant default mode processing (Lustig et al., 2003; Grady et al., 2006; Miller et al., 2008).

One interpretation of the coupling of activity in PFC with activity in auditory areas during failed encoding is that increased PFC activity reflects increased inhibitory input from this region (Jonides et al., 2000) in an attempt to counteract the distraction of irrelevant auditory input. Conversely, it is possible that this pattern of connectivity reflects the misallocation of attention to the distracting auditory input leading to failed encoding attempts. If the former were true, one might expect increased activity in this PFC region during successful encoding trials when interference is overcome or inhibited, relative to failed encoding trials. However we observed the opposite, i.e., increased activation for failed encoding attempts, supporting the latter hypothesis. These hypotheses cannot be conclusively resolved in the current data, but suggest directions for future investigation. 
The pattern of increased activity associated with subsequently forgotten faces in older adults is probably not simply due to the fact that they fail to recognize more faces than do the young adults. Support for this idea was found in the contrast of young adults with fewer misses to those with a greater number of misses (levels that were comparable with older adults). This analysis showed that low-performing younger adults did not have the same pattern of brain activity for forgotten faces seen in the older adults, suggesting that the effect of distraction is age-related and not performance related.

In conclusion, our results provide new insight into the nature of age-related memory decline, suggesting that at least two distinct neurocognitive mechanisms play a role in encoding failures in older adults: (1) a general failure to engage brain regions crucial to encoding new information, such as MTL structures, can contribute to failed encoding regardless of age. (2) Older, but not younger, adults are vulnerable to distraction due to an inability to suppress processing of irrelevant environmental stimuli. This distraction is reflected in age differences in those brain areas that process the irrelevant stimuli, such as auditory stimuli during a visual task (i.e., auditory cortices), as well as in DMRs (e.g., medial parietal cortex) that may be involved in environmental monitoring. In addition, our results support previous research showing that while both younger and older adults' performance was decreased on a long-term recognition task following encoding inside, relative to outside, the fMRI scanner, the deleterious effects of fMRI on performance were greater for older adults (Gutchess and Park, 2006). This raises the possibility that the distracting properties of fMRI scanning per se may be disproportionately detrimental to older adults, relative to younger adults, a potential confound that may have implications for fMRI investigations of neurocognitive aging.

\section{References}

Bartlett JC, Leslie JE, Tubbs A, Fulton A (1989) Aging and memory for pictures of faces. Psychology and Aging 4:276-283.

Brandt J (1991) The Hopkins Verbal Learning Test: Development of a new memory test with six equivalent forms. Clin Neuropsychol 5:125-142.

Brewer JB, Zhao Z, Desmond JE, Glover GH, Gabrieli JDE (1998) Making memories: brain activity that predicts how well visual experience will be remembered. Science 281:1185-1187.

Connelly SL, Hasher L, Zacks RT (1991) Age and reading: the impact of distraction. Psychol Aging 6:533-541.

Cox RW, Hyde JS (1997) Software tools for analysis and visualization of fMRI data. NMR in biomedicine 10:171-178.

Craik FIM, McDowd JM (1987) Age differences in recall and recognition. J Exp Psychol Learn Mem Cogn 13:474-479.

Daselaar SM, Veltman DJ, Rombouts SA, Raaijmakers JG, Jonker C (2003) Neuroanatomical correlates of episodic encoding and retrieval in young and elderly subjects. Brain 126:43-56.

Efron B, Tibshirani R (1986) Bootstrap methods for standard errors, confidence intervals and other measures of statistical accuracy. Stat Sci 1:54-77.

Fabiani M, Low KA, Wee E, Sable JJ, Gratton G (2006) Reduced suppression or labile memory? Mechanisms of inefficient filtering of irrelevant information in older adults. J Cogn Neurosci 18:637-650.

Folstein MF, Folstein SE, McHugh PR (1975) "Mini Mental State"- a practical method for grading the cognitive state of patients for the clinician. J Psychiatr Res 12:189-198.

Friston K, Holmes A, Worsley K, Poline J, Frith C, Frackowiak R (1995)
Statistical parametric maps in functional imaging: a general linear approach. Hum Brain Mapp 2:189-210.

Gazzaley A, Cooney JW, Rissman J, D’Esposito M (2005) Top-down suppression deficit underlies working memory impairment in normal aging. Nat Neurosci 8:1298-1300.

Gilbert SJ, Dumontheil I, Simons JS, Frith CD, Burgess PW (2007) Comment on "Wandering minds: the default network and stimulusindependent throught". Science 317:43.

Grady CL, McIntosh AR, Horwitz B, Maisog JM, Ungerleider LG, Mentis MJ, Pietrini P, Schapiro MB, Haxby JV (1995) Age-related reductions in human recognition memory due to impaired encoding. Science 269:218-221.

Grady CL, McIntosh AR, Craik FI (2003) Age-Related differences in the functional connectivity of the hippocampus during memory encoding. Hippocampus 13:572-586.

Grady CL, Springer MV, Hongwanishkul D, McIntosh AR, Winocur G (2006) Age-related changes in brain activity across the adult lifespan. J Cogn Neurosci 18:227-241.

Grady CL, Yu H, Alain C (2008) Age-related differences in brain activity underlying working memory for spatial and nonspatial auditory information. Cereb Cortex 18:189-199.

Gusnard DA, Akbudak E, Shulman GL, Raichle ME (2001) Medial prefrontal cortex and self-referential mental activity: relation to a default mode of brain function. Proc Natl Acad Sci U S A 98:4259-4264.

Gutchess AH, Park DC (2006) fMRI environment can impair memory performance in young and elderly adults. Brain Res 1099:133-140.

Haxby JV, Ungerleider LG, Horwitz B, Maisog JM, Rapoport SI, Grady CL (1996) Face encoding and recognition in the human brain. Proc Natl Acad Sci U S A 93:922-927.

Healey MK, Campbell KL, Hasher L (2008) Cognitive aging and increased distractibility: costs and potential benefits. In: Progress in brain Research, Vol 169: The essence of memory (Sossin W, Lacaille JC, Castellucci VF, Bellebille S, ed). Amsterdam: Elsevier.

Jonides J, Marshuetz C, Smith EE, Reuter-Lorenz PA, Koeppe RA, Hartley A (2000) Age differences in behavior and PET activation reveal differences in interference resolution in verbal working memory. J Cogn Neurosci 12:188-196.

Lustig C, Snyder AZ, Bhakta M, O’Brien KC, McAvoy M, Raichle ME, Morris JC, Buckner RL (2003) Functional deactivations: change with age and dementia of the Alzheimer type. Proc Natl Acad Sci U S A 100:14504-14509.

Lustig C, Hasher L, Tonev ST (2006) Distraction as a determinant of processing speed. Psychonomic bulletin and review 13:619-625.

McIntosh AR (1999) Mapping cognition to the brain through neural interactions. Memory 7:523-548.

McIntosh AR, Bookstein FL, Haxby JV, Grady CL (1996) Spatial pattern analysis of functional brain images using Partial Least Squares. Neuroimage 3:143-157.

McIntosh AR, Chau WK, Protzner AB (2004) Spatiotemporal analysis of event-related fMRI data using partial least squares. NeuroImage 23:764-775.

Miller SL, Celone K, DePeau K, Diamond E, Dickerson BC, Rentz D, Pihlajamäki M, Sperling RA (2008) Age-related memory impairment associated with loss of parietal deactivation but preservd hippocampal activation. Proc Natl Acad Sci U S A 105:2181-2186.

Morcom AM, Good CD, Frackowiak RS, Rugg MD (2003) Age effects on the neural correlates of successful memory encoding. Brain 126:213-229.

Raichle ME, Snyder AZ (2007) A default mode of brain function: a brief history of an evolving idea. Neuroimage 37:1083-1090; discussion 1097-1089.

Raven JC, Court JH, Raven J (1988) The Mill Hill Vocabulary Scale: 1988 revision. London: H.K. Lewis.

Sampson PD, Streissguth AP, Barr HM, Bookstein FL (1989) Neurobehavioral effects of prenatal alchohol: Part II. Partial least squares analysis. Neurotoxicol Teratol 11:477-491. 